\title{
Very Low Frequency of PAD in People with CHD in Six Middle Eastern Countries
}

\author{
Hasan Kutsi Kabul ${ }^{1}$ and Ilker Tasci ${ }^{2}$ \\ ${ }^{1}$ Department of Cardiology, Gulhane School of Medicine, 06018 Ankara, Turkey \\ ${ }^{2}$ Department of Internal Medicine and Geriatrics, Gulhane School of Medicine, 06018 Ankara, Turkey
}

Correspondence should be addressed to Ilker Tasci, itasci@gata.edu.tr

Received 6 January 2012; Accepted 24 January 2012

Copyright (c) 2012 H. K. Kabul and I. Tasci. This is an open access article distributed under the Creative Commons Attribution License, which permits unrestricted use, distribution, and reproduction in any medium, provided the original work is properly cited.

We read with interest the article by Al-Thani et al. [1] reporting a very low frequency $(2.6 \%)$ of peripheral arterial disease (PAD) in people with acute coronary syndrome in a multicenter study conducted in six Middle Eastern countries. A recent multicenter study in Turkey showed 20\% prevalence of PAD in people with established cardiovascular disease or who were at risk for atherosclerotic diseases [2].

Diabetes that was present in $70 \%$ of the participants in their study is actually a major cause of arterial stiffness or calcification, which frequently results in misclassification of diabetics in ankle brachial index (ABI) testing by causing paradoxically high values [3]. In this context, it would be utmost helpful if the authors could provide some data related to the prevalence of a high ABI $(>1.4)$ in their study population, so that the readers could recognize whether a high ABI potentially influenced the frequency of a low ABI.

Another point that should be noted is the method of assessment of PAD by ABI testing. In recent years, many societies published detailed consensus guidelines on the definition and management of PAD [4-6]. Collectively, the ABI cut-off value for the diagnosis of PAD is currently accepted as $\leq 0.9$. Although ABI of $<0.8$ can be found in several surveys as the diagnostic threshold, adherence to guidelines and their updates would be a superior evidencebased approach. In such case, it could be speculated that the prevalence of PAD would be recorded some higher in the study by Al-Thani et al.

A final concern is the need for a correct calculation of ABI. As explained clearly in the TASC II guidelines, [4] higher readings of dorsalis pedis or tibialis posterior arteries should be used as the numerator in the index formula. However, Al-Thani et al. used the average (i.e., mean of dorsalis pedis plus tibialis posterior records) systolic blood pressure readings in each ankle as the numerator in order to calculate the ABI ratio. Compared to guideline recommendations, this method obviously results in use of lower values for any ankle especially when one of the readings is lower than the other, causing an increase in the frequency of a low ABI. Opposite to our concern regarding the ABI cut-off in the previous section, such a calculation could further reduce the reported prevalence of PAD in this multicenter large scale survey. However, through a scientific view, this does not eliminate the potential limitations. We guess that the readers would appreciate if the authors could comment on these issues.

\section{References}

[1] H. A. Al-Thani, A. El-Menyar, M. Zubaid et al., "Peripheral arterial disease in patients presenting with acute coronary syndrome in six middle eastern countries," International Journal of Vascular Medicine, vol. 2011, Article ID 815902, 8 pages, 2011.

[2] A. K. Bozkurt, I. Tasci, O. Tabak, M. Gumus, and Y. Kaplan, "Peripheral artery disease assessed by ankle-brachial index in patients with established cardiovascular disease or at least one risk factor for atherothrombosis-CAREFUL study: a national, multi-center, cross-sectional observational study," BMC Cardiovascular Disorders, vol. 11, article 4, 2011.

[3] L. Potier, C. Abi Khalil, K. Mohammedi, and R. Roussel, "Use and utility of ankle brachial index in patients with diabetes," EJVES Extra, vol. 41, no. 1, pp. 110-116, 2011.

[4] L. Norgren, W. R. Hiatt, J. A. Dormandy, M. R. Nehler, K. A. Harris, and F. G. R. Fowkes, "Inter-society consensus for the management of peripheral arterial disease (TASC II)," Journal of Vascular Surgery, vol. 45, no. 1, supplement, pp. S5-S67, 2007. 
[5] T. W. Rooke, A. T. Hirsch, S. Misra et al., "2011 ACCF/AHA focused update of the guideline for the management of patients with peripheral artery disease (updating the 2005 guideline) a report of the American college of cardiology foundation/American heart association task force on practice guidelines," Journal of the American College of Cardiology, vol. 58, pp. 2020-2045, 2011.

[6] M. Tendera, V. Aboyans, M. L. Bartelink et al., "ESC Guidelines on the diagnosis and treatment of peripheral artery diseases: document covering atherosclerotic disease of extracranial carotid and vertebral, mesenteric, renal, upper and lower extremity arteries," European Heart Journal, vol. 32, no. 22, pp. 2851-2906, 2011. 


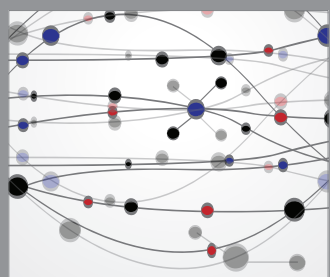

The Scientific World Journal
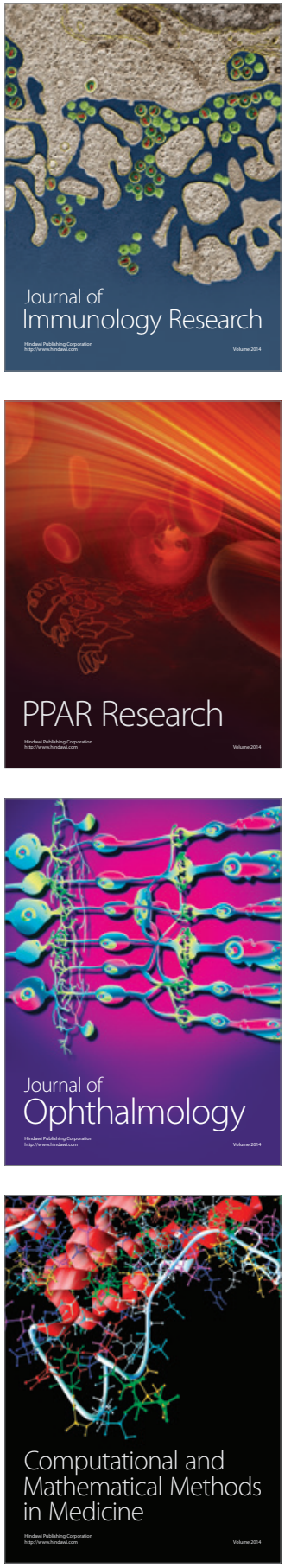

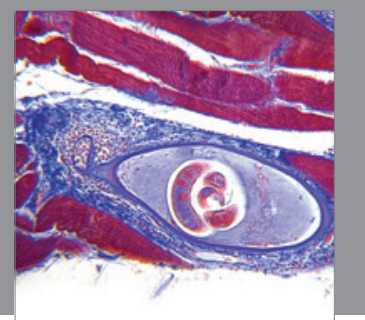

Gastroenterology

Research and Practice
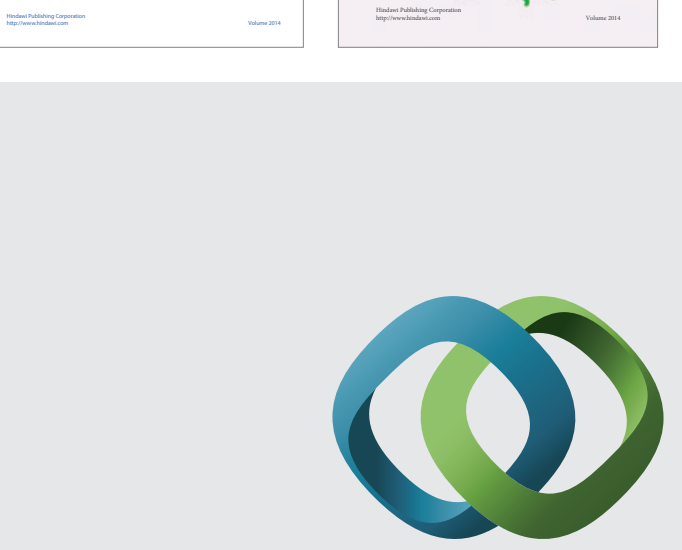

\section{Hindawi}

Submit your manuscripts at

http://www.hindawi.com
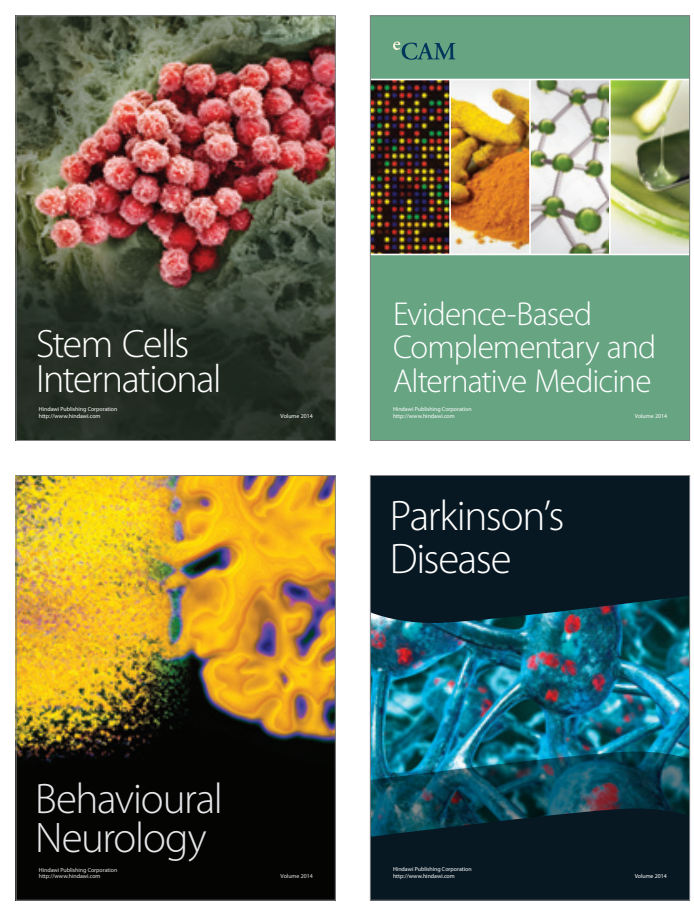

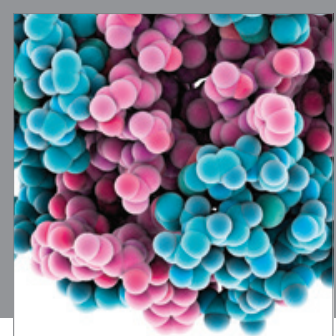

Journal of
Diabetes Research

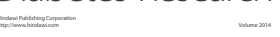

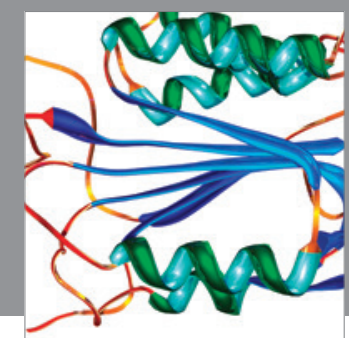

Disease Markers
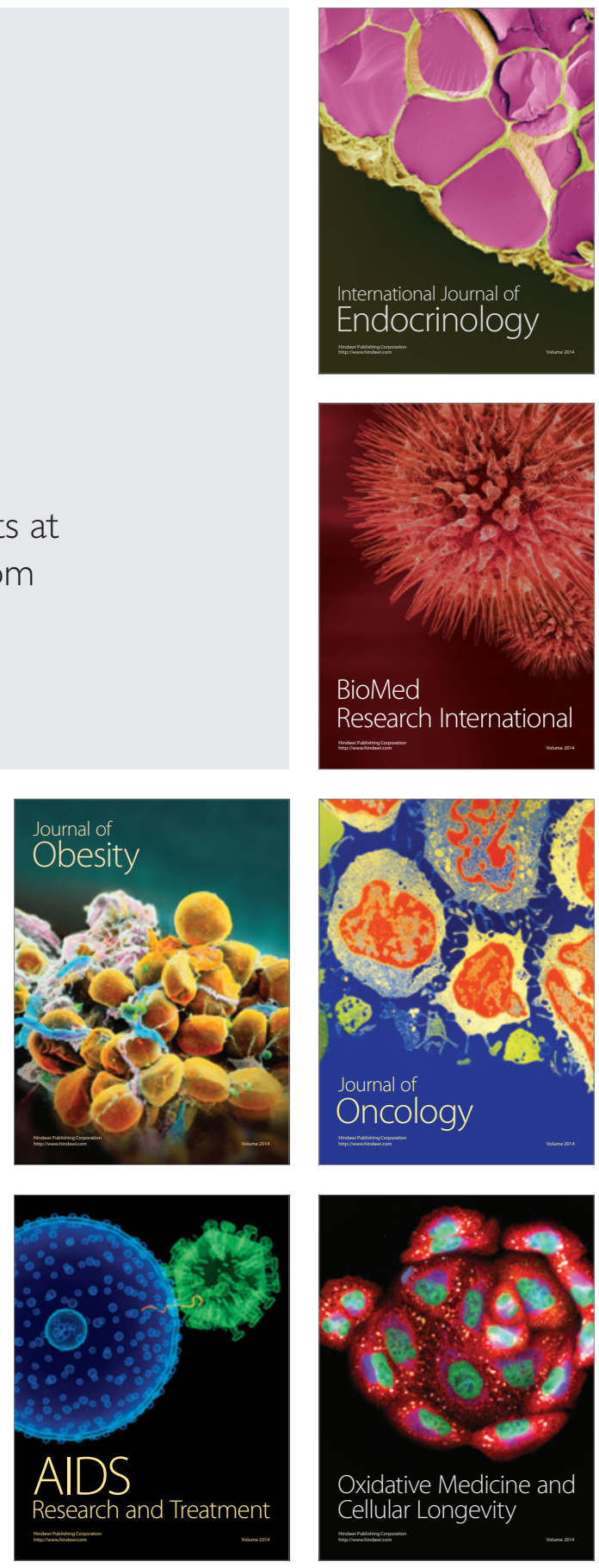